\title{
Some new results on integration for multifunction
}

\author{
Domenico Candeloro' $^{1}$ (D) Luisa Di Piazza ${ }^{2}$. \\ Kazimierz Musial ${ }^{3}$ (D) Anna Rita Sambucini ${ }^{1}$
}

\begin{abstract}
It has been proven in Di Piazza and Musiał (Set Valued Anal 13:167-179, 2005, Vector measures, integration and related topics, Birkhauser Verlag, Basel, vol 201, pp 171-182, 2010) that each Henstock-Kurzweil-Pettis integrable multifunction with weakly compact values can be represented as a sum of one of its selections and a Pettis integrable multifunction. We prove here that if the initial multifunction is also Bochner measurable and has absolutely continuous variational measure of its integral, then it is a sum of a strongly measurable selection and of a variationally Henstock integrable multifunction that is also Birkhoff integrable (Theorem 3.4). Moreover,
\end{abstract}

Dedicated to Prof. Hans Weber on the occasion of his 70th birthday with deep esteem.

Communicated by P. De Lucia.

This research was supported by the Grant Prot. No. U2015/001379 of GNAMPA - INDAM (Italy); by University of Perugia_Dept. of Mathematics and Computer Sciences_-Grant No. 2010.011.0403 and by University of Palermo (Italy).

$凶 \quad$ Luisa Di Piazza

luisa.dipiazza@unipa.it

Domenico Candeloro

domenico.candeloro@unipg.it

Kazimierz Musiał

musial@math.uni.wroc.pl

Anna Rita Sambucini

anna.sambucini@unipg.it

1 Department of Mathematics and Computer Sciences, 06123 Perugia, Italy

2 Department of Mathematics, University of Palermo, Via Archirafi 34, 90123 Palermo, Italy

3 Institut of Mathematics, Wrocław University, Pl. Grunwaldzki 2/4, 50-384 Wrocław, Poland 
in case of strongly measurable (multi)functions, a characterization of the Birkhoff integrability is given using a kind of Birkhoff strong property.

Keywords Multifunction · Set-valued Pettis integral · Set-valued variationally Henstock and Birkhoff integrals · Selection

Mathematics Subject Classification 28B20 - 26E25 - 26A39 - 28B05 - 46G10 . 54C60 - 54C65

\section{Introduction}

Integration of vector valued functions is strongly motivated by general problems of modern analysis including control theory, economics and differential inclusions. In many situations it is enough to use the well-known Bochner and Pettis integrals involved with the classical Lebesgue theory. In the last decades however gauge (nonabsolute) integrals have been also considered [2-4, 7, 8, 10-14, 18, 19, 22, 24, 25, 27, 29] after the pioneering studies of G. Birkhoff, J. Kurzweil, R. Henstock and E. J. McShane. The Birkhoff integral was introduced in [1] and recently investigated in $[9,15,16,31,39,40]$. B. Cascales, V. M. Kadets, M. Potyrala, J. Rodriguez, and other authors considered the unconditional Riemann-Lebesgue multivalued integral.

This article is organized in the following manner. In Sect. 2 we give some preliminaries and the definitions. In Sect. 3 a new decomposition theorem for Bochner measurable and weakly compact valued Henstock-Kurzweil-Pettis integrable multifunction $\Gamma$ with absolutely continuous variational measure of its integral is obtained, involving Birkhoff and variationally Henstock integrability of the multifunction $G$ such that $\Gamma=f+G$. It is also shown that the conditions in Theorem 3.4 do not imply, in general, the variational integrability of the multifunction $\Gamma$ and an example is given (Example 3.6).

Finally, in case of strongly measurable (multi)functions, a characterization of the Birkhoff integrability is given using a kind of Birkhoff strong property (see Definition 3.11).

\section{Preliminary facts}

Throughout $[0,1]$ is the unit interval of the real line equipped with the usual topology and Lebesgue measure $\lambda, \mathcal{L}$ denotes the family of all Lebesgue measurable subsets of $[0,1]$, and $\mathcal{I}$ is the collection of all closed subintervals of [0,1]: if $I \in \mathcal{I}$ then its Lebesgue measure will be denoted by $|I|$.

$X$ is an arbitrary Banach space with its dual $X^{*}$. The closed unit ball of $X^{*}$ is denoted by $B\left(X^{*}\right)$. Following the notation in [17], $c w k(X)$ is the family of all nonempty convex weakly compact subsets of $X$ and $c k(X)$ is the family of all compact members of $c w k(X)$. We consider on $c w k(X)$ the Minkowski addition $(A+B:=$ $\{a+b: a \in A, b \in B\})$ and the standard multiplication by scalars. $d_{H}$ is the Hausdorff distance in $c w k(X)$ and $c w k(X)$ with this metric is a complete metric space. For every $A \in \operatorname{cwk}(X),\|A\|:=d_{H}(A,\{0\})=\sup \{\|x\|: x \in A\}$. 
For every $C \in \operatorname{cwk}(X)$ the support function of $C$ is denoted by $s(\cdot, C)$ and defined on $X^{*}$ by $s\left(x^{*}, C\right):=\sup \left\{\left\langle x^{*}, x\right\rangle: x \in C\right\}$, for each $x^{*} \in X^{*}$.

A map $\Gamma:[0,1] \rightarrow 2^{X} \backslash\{\emptyset\}$ (= non-empty subsets of $\left.X\right)$ is called a multifunction.

A multifunction $\Gamma:[0,1] \rightarrow c w k(X)$ is said to be scalarly measurable if for every $x^{*} \in X^{*}$, the map $s\left(x^{*}, \Gamma(\cdot)\right)$ is measurable.

$\Gamma$ is said to be Bochner measurable if there exists a sequence of simple multifunctions $\Gamma_{n}:[0,1] \rightarrow \operatorname{cwk}(X)$ such that

$$
\lim _{n \rightarrow \infty} d_{H}\left(\Gamma_{n}(t), \Gamma(t)\right)=0
$$

for almost all $t \in[0,1]$.

A function $f:[0,1] \rightarrow X$ is called a selection of $\Gamma$ if $f(t) \in \Gamma(t)$, for every $t \in[0,1]$.

A partition $\mathcal{P}$ in $[0,1]$ is a collection $\left\{\left(I_{1}, t_{1}\right), \ldots,\left(I_{p}, t_{p}\right)\right\}$, where $I_{1}, \ldots, I_{p}$ are nonoverlapping subintervals of $[0,1], t_{i}$ is a point of $[0,1], i=1, \ldots, p$.

If $\cup_{i=1}^{p} I_{i}=[0,1]$, then $\mathcal{P}$ is a partition of $[0,1]$. If $t_{i}$ is a point of $I_{i}, i=1, \ldots, p$, we say that $\mathcal{P}$ is a Perron partition of $[0,1]$.

A gauge on $[0,1]$ is a positive function on $[0,1]$. For a given gauge $\delta$ on $[0,1]$, we say that a partition $\left\{\left(I_{1}, t_{1}\right), \ldots,\left(I_{p}, t_{p}\right)\right\}$ is $\delta$-fine if $I_{i} \subset\left(t_{i}-\delta\left(t_{i}\right), t_{i}+\delta\left(t_{i}\right)\right), i=$ $1, \ldots, p$.

We recall that an interval multifunction $\Phi: \mathcal{I} \rightarrow c w k(X)$ is said to be finitely additive if for every non-overlapping intervals $I_{1}, I_{2} \in \mathcal{I}$ such that $I_{1} \cup I_{2} \in \mathcal{I}$ we have $\Phi\left(I_{1} \cup I_{2}\right)=\Phi\left(I_{1}\right)+\Phi\left(I_{2}\right)$. $\Phi$ is then called an interval multimeasure.

A multifunction $M: \mathcal{L} \rightarrow c w k(X)$ is said to be a multimeasure if for every $x^{*} \in X^{*}$, the map $A \mapsto s\left(x^{*}, M(A)\right)$ is a real-valued measure (see [28, Theorem 8.4.10]). We say that the multimeasure $M: \mathcal{L} \rightarrow c w k(X)$ is $\lambda$-continuous and we write $M \ll \lambda$, if $\lambda(A)=0$ yields $M(A)=\{0\}$.

For the definitions of Birkhoff, Henstock, McShane and variationally Henstock $\operatorname{cwk}(X)$-valued multifunctions we refer to [11, Definitions 2.2., 2.3, 2.8].

If $X=\mathbb{R}$ and $\Gamma$ is a function, we speak of Henstock-Kurzweil integrability instead of Henstock one. Each of these integrals turns out to be an additive mapping, on $\mathcal{I}$ or $\mathcal{L}$ respectively, and is called primitive, or also integral measure of the mapping $\Gamma$. From the definition and the completeness of the Hausdorff metric the $c w k(X)$-valued integrals defined before have weakly compact values.

Definition 2.1 A multifunction $\Gamma:[0,1] \rightarrow c w k(X)$ is said to be Pettis (resp. Henstock-Kurzweil-Pettis integrable (in short (HKP)-integrable)) if $s\left(x^{*}, \Gamma\right)$ is integrable (resp. Henstock-Kurzweil integrable) for each $x^{*} \in X^{*}$, and for each $A \in \mathcal{L}$ (resp. $I \in \mathcal{I}$ ) there exists an element $w_{A} \in \operatorname{cwk}(X)\left(\operatorname{resp} . w_{I} \in \operatorname{cwk}(X)\right)$ such that $x^{*}\left(w_{A}\right)=\int_{A} s\left(x^{*}, \Gamma(t)\right) d t$ (resp. $\left.x^{*}\left(w_{I}\right)=(H) \int_{I} s\left(x^{*}, \Gamma(t)\right) d t\right)$ holds, for each $x^{*} \in X^{*}$.

There is a large literature concerning Pettis and Henstock-Kurzweil-Pettis integral for functions and multifunctions; we refer the reader to [15,21,22,32-35,37].

It follows from the classical properties of the Henstock-Kurzweil integral that the primitives of Henstock or $(H K P)$ integrable multifunctions are interval multimeasures, 
while the primitives of Pettis or Birkoff integrable multifunctions are multimeasures on the $\sigma$-algebra $\mathcal{L}$ (see [16, Theorem 4.1(i)]).

Moreover, as observed in [11], the multivalued integral of a Pettis integrable $c w k(X)$-valued multifunction is absolutely continuous with respect to $\lambda$ and its embedded measure is an $l_{\infty}\left(B\left(X^{*}\right)\right)$-valued countably additive measure.

For the definition of the variational measure $V_{\Phi}$ associated to a finitely additive interval measure $\Phi: \mathcal{I} \rightarrow \mathbb{R}$ we refer the reader to [6,23]. In particular, we recall that the variational measure $V_{\phi}$ of the primitive $\phi$ of a variationally Henstock integrable mapping is a (possibly unbounded) $\lambda$-continuous measure on $\mathcal{L}$ : see $[38$, Proposition 3.3.1].

Finally, a useful tool to study the $c w k(X)$-valued multifunctions is the Rådström embedding (see for example [30]) given by $i(A):=s(\cdot, A)$. It satisfies the following properties:

(1) $i(\alpha A+\beta C)=\alpha i(A)+\beta i(C)$ for every $A, C \in c w k(X), \alpha, \beta \in \mathbb{R}^{+}$;

(2) $d_{H}(A, C)=\|i(A)-i(C)\|_{\infty}, \quad A, C \in \operatorname{cwk}(X)$;

(3) $i(c w k(X))=\overline{i(c w k(X))}$ (norm closure).

Observe that it follows directly from the definitions that a multifunction $\Gamma$ : $[0,1] \rightarrow c w k(X)$ is Birkhoff (resp. Henstock, McShane, variationally Henstock) integrable if and only if $i(\Gamma)$ is integrable in the same sense.

\section{Variationally Henstock integrable selections}

Concerning existence of selections of gauge integrable mappings with values in $c w k(X)$, several results have been obtained recently in $[4,10,11,21,22,39]$. The purpose of this paper is to revisit the last results, trying to generalize it to a more general case. We begin with some useful Lemmas.

Lemma 3.1 Let $\Gamma:[0,1] \rightarrow c w k(X)$ be any $(H K P)$-integrable multifunction, and let $f:[0,1] \rightarrow X$ be any $(H K P)$-integrable selection of $\Gamma$. Then, if $\phi$ and $\Phi$ denote respectively the primitives of $f$ and $\Gamma$, it is

$$
\phi(I) \in \Phi(I)
$$

for every subinterval $I \subset[0,1]$.

Proof It can be deduced as in the proof of [10, Proposition 2.7].

Lemma 3.2 Let $\Gamma:[0,1] \rightarrow c w k(X)$ be any $(H K P)$-integrable multifunction, and let $f$ be any $(H K P)$-integrable selection of $\Gamma$. If the variational measure $V_{\Phi}$ associated to the primitive $\Phi$ of $\Gamma$ is absolutely continuous with respect to the Lebesgue measure $\lambda$, the same holds for the variational measure of the primitive $\phi$ of $f$. In particular this holds whenever $\Gamma$ is variationally Henstock integrable.

Proof From the previous Lemma we have that $\phi(I) \in \Phi(I)$ for every interval $I \subset$ $[0,1]$. Then $V_{\phi} \leq V_{\Phi}$. Since $V_{\Phi}$ is $\lambda$-continuous the same holds for $V_{\phi}$, of course. 
A crucial tool is the following.

Theorem 3.3 Let $G:[0,1] \rightarrow c w k(X)$ be any McShane integrable mapping. Then $G$ is variationally Henstock integrable if and only if it is Bochner measurable and the variational measure of its integral is $\lambda$-continuous.

Proof If $G$ is McShane integrable, then also $i(G)$ is McShane, hence Pettis, integrable and we may apply [6, Lemma 4.1].

Here is the new decomposition theorem which extends [11, Theorem 5.1].

Theorem 3.4 Let $\Gamma:[0,1] \rightarrow \operatorname{cwk}(X)$ satisfy the following conditions:

(3.4.1) $\Gamma$ is Bochner measurable;

(3.4.2) $\Gamma$ is $H K$ P-integrable;

(3.4.3) the variational measure associated to the primitive of $\Gamma$ is $\lambda$-continuous.

Then $\Gamma$ can be decomposed as the sum $\Gamma=f+G$, where $f$ is any strongly measurable selection of $\Gamma$ and $G$ is a variationally Henstock and Birkhoff integrable multifunction.

Proof Let $f$ be any Bochner measurable selection of $\Gamma$. Then $f$ is $H K P$-integrable and the mapping $G$ defined by $\Gamma=G+f$ is Pettis integrable (see [21, Theorem 1]). Moreover $i(G)$ and $G$ are strongly measurable. By [16, Proposition 4.5] $i(G)$ is Pettis integrable, since the range of $i(G)$ is separable. So, by [37, Corollary 5.11] $i(G)$ is McShane and so $G$ is McShane integrable.

By (3.4.3) and by Lemma 3.2 the variational measure $V_{\phi}$ associated to $f$ is $\lambda$-continuous and so the variational measure associated to the primitive of $G$ is $\lambda$ continuous too. So, the mapping $G$ is variationally Henstock integrable thanks to Theorem 3.3. Birkhoff integrability of $G$ follows from [10, Proposition 4.1] since the support functionals of $G$ are non-negative.

Remark 3.5 We observe that the conditions (3.4.1), (3.4.2), (3.4.3) do not imply in general variational Henstock integrability of $\Gamma$. But the implication holds true, as already observed, if (3.4.2) is replaced by the stronger request that $\Gamma$ is Pettis integrable, by Theorem 3.3.

So, we shall now give an example of a mapping $f:[0,1] \rightarrow c_{0}$, satisfying (3.4.1), (3.4.2), (3.4.3), but not Henstock integrable (and so, a fortiori, not variationally Henstock integrable). The example is the same as [5, Example 2], and [26, Example].

Example 3.6 Fix any disjoint sequence of closed subintervals $\left(J_{n}\right)_{n}:=\left(\left[a_{n}, b_{n}\right]\right)_{n}$ in $[0,1]$, such that $0=a_{1}<b_{1}<a_{2}<b_{2}<\cdots$ and $\lim _{n} b_{n}=1$.

Now the function $f$ is defined as follows:

$$
f_{n}(t):=\frac{1}{2}\left|J_{2 n-1}\right| \chi_{J_{2 n-1}}(t)-\frac{1}{2}\left|J_{2 n}\right| \chi_{J_{2 n}}(t),
$$


for each $t \in[0,1]$, where $f_{n}$ denotes the $n$th component of $f$. Proceeding as in the quoted papers $[5,26]$, it is possible to see that $f$ is (HKP)-integrable, and, for every interval $I \subset[0,1]$ :

$$
\int_{I} f d t=\left(\frac{\lambda\left(I \cap J_{2 n-1}\right)}{2\left|J_{2 n-1}\right|}-\frac{\lambda\left(I \cap J_{2 n}\right)}{2\left|J_{2 n}\right|}\right)_{n} .
$$

However, as observed in [5, Example 2], the set $\left\{\int_{I} f(t) d t: I \in \mathcal{I}\right\}$ is not relatively norm compact in $c_{0}$, hence $f$ is not Henstock integrable, thanks to [5, Proposition 1].

Since $c_{0}$ is separable, $f$ is strongly measurable; then, we only have to prove that the variational measure associated with the integral $\Gamma$ of $f$ is $\lambda$-continuous.

To this aim, let us fix any null set $A \in \mathcal{L}$, and fix any $\varepsilon>0$. Then clearly $\lambda\left(A \cap J_{n}\right)=$ 0 for every integer $n$. So, for every $n$ there exists an open set $U_{n}$ such that $A \cap J_{n} \subset U_{n}$ and $\lambda\left(U_{n}\right) \leq \frac{\varepsilon}{2^{n}}\left|J_{n}\right|$.

Now, for each element $t \in A$, we define $\delta(t)$ in such a way that $[t-\delta(t), t+\delta(t)] \subset$ $U_{n}$ whenever $t \in A \cap J_{n}^{0}$, and $\left.[t-\delta(t), t+\delta(t)] \subset\right] b_{n(t)}, a_{n(t)+1}\left[\right.$ as soon as $t \notin \cup_{n} J_{n}$ and $n(t)$ is such that $t \in] b_{n(t)}, a_{n(t)+1}$ [. Finally, if $t \in A$ is one of the points $a_{n}$ or $b_{n}$, we choose $\delta(t)$ in such a way that $[t-\delta(t), t+\delta(t)] \subset U_{n}$ and $[t-\delta(t), t+\delta(t)]$ intersects just one of the intervals $J_{n}$.

So, if $\left\{\left(I_{k}, t_{k}\right), k=1, \ldots, K\right\}$ is any $\delta$-fine Henstock partition in $[0,1]$, with tags in $A$, for each index $k$ the interval $I_{k}$ intersects at most one of the intervals $J_{n}$, and therefore $F\left(I_{k}\right):=\int_{I_{k}} f(t) d t$ has no more than one component different from 0 (say $n)$, and

$$
\left\|\int_{I_{k}} f(t) d t\right\| \leq \frac{\lambda\left(I_{k} \cap J_{n}\right)}{2\left|J_{n}\right|} .
$$

Hence, summing as $k=1, \ldots, K$, we get

$$
\begin{aligned}
\sum_{k}\left\|F\left(I_{k}\right)\right\| & =\sum_{n} \sum_{t_{k} \in J_{n}}\left\|\int_{I_{k}} f(t) d t\right\| \leq \sum_{n} \sum_{t_{k} \in J_{n}} \frac{\lambda\left(I_{k} \cap J_{n}\right)}{2\left|J_{n}\right|} \leq \\
& \leq \sum_{n} \frac{\lambda\left(U_{n}\right)}{2\left|J_{n}\right|} \leq \sum_{n} \frac{\varepsilon}{2^{n}}=\varepsilon .
\end{aligned}
$$

This proves that $f$ satisfies condition (3.4.3) as required.

The function from the above example has a trivial decomposition: $f(t)=\{0\}+$ $f(t)$. To have an example of a multifunction $\Gamma:[0,1] \rightarrow c w k\left(c_{0}\right)$ satisfying (3.4.1), (3.4.2), (3.4.3) and possessing a non-trivial decomposition, it is sufficient to take any variationally Henstock integrable multifunction $\Gamma_{0}:[0,1] \rightarrow c w k\left(c_{0}\right)$, and define: $\Gamma(t)=\Gamma_{0}(t)+f(t)$, for $t \in[0,1]$, where $f$ is the function defined above. Since $i(\Gamma)=i\left(\Gamma_{0}\right)+i(\{f(t)\})$, we easily see that $i(\Gamma)$ (and so $\Gamma$ ) satisfies (3.4.1), (3.4.2), (3.4.3), but it cannot be Henstock integrable, otherwise $i(\{f\})$ (and therefore $f$ ) would be, by difference.

As a consequence of Theorem 3.4 we can obtain the following corollary which appears also in [11, Thorem 5.3] with a different proof. 
Corollary 3.7 Let $\Gamma:[0,1] \rightarrow c w k(X)$ be any variationally Henstock integrable multifunction. Then every strongly measurable selection $f$ of $\Gamma$ is variationally Henstock integrable and $\Gamma=G+f$, where $G$ is Birkhoff and variationally Henstock integrable.

Proof Since $\Gamma$ is variationally Henstock integrable, it satisfies (3.4.1), (3.4.2) and (3.4.3), so from Theorem 3.4 we deduce that, for every strongly measurable selection $f$ the mapping $G$ defined by $\Gamma=G+f$ is variationally Henstock and Birkhoff integrable. Finally, since $i(\{f\})=i(\Gamma)-i(G)$, it follows easily that $i(\{f\})$ (and therefore also $f$ ) is variationally Henstock integrable.

We have cited before [37, Corollary 5.11] which ensures that for strongly measurable functions, the Pettis and the Birkhoff integrability are the same. We shall now present one more characterization of the Birkhoff integrability for strongly measurable integrands.

Proposition 3.8 Assume that $f:[0,1] \rightarrow X$ is strongly measurable. Then for every $\varepsilon>0$ there exists a countable partition of $[0,1]$, made of measurable sets $\left(A_{h}\right)_{h}$, such that $\left.f\right|_{A_{h}}$ is Bochner integrable for every $h \in \mathbb{N}$ and

$$
\sum_{k}\left\|f\left(t_{k}\right) \lambda\left(A_{k}^{\prime}\right)-\int_{A_{k}^{\prime}} f\right\| \leq \varepsilon
$$

holds true, for any partition $\left\{A_{k}^{\prime}\right\}_{k}$ refining $\left(A_{n}\right)_{n}$, and any choice of points $t_{k}$ in $A_{k}^{\prime}$, $k \in \mathbb{N}$.

Proof Since $f$ is Bochner measurable, then $f$ is also Lusin measurable, see [36, Section 3]. Now, in order to prove (1), fix arbitrarily $\varepsilon>0$. Thanks to Lusin measurability of $f$ there exists a sequence $\left(B_{n}\right)_{n}$ of pairwise disjoint closed subsets of $[0,1]$, such that $\sum_{n=1}^{+\infty} \lambda\left(B_{n}\right)=1$, and a corresponding sequence $\left(\delta_{n}\right)_{n}$ of positive real numbers, such that

$$
\left\|f(t)-f\left(t^{\prime}\right)\right\| \leq \varepsilon
$$

holds for every $n$, as soon as $t, t^{\prime}$ are in $B_{n}$ and $\left|t-t^{\prime}\right| \leq \delta_{n}$. Since $f_{\mid B_{n}}$ is continuous, it is obviously Bochner integrable in $B_{n}$, hence we can deduce also that

$$
\left\|f(t)-\frac{\int_{B_{n} \cap I} f}{\lambda\left(B_{n} \cap I\right)}\right\| \leq \varepsilon
$$

holds true, for every $n$, every interval $I \subset[0,1]$ with $|I| \leq \delta_{n}$ and every point $t \in B_{n} \cap I$ (provided the latter is non-negligible).

Now, in order to construct the announced partition $\left(A_{n}\right)_{n}$, for every integer $n$ fix a finite partition $\left\{I_{1}^{n}, \ldots, I_{N(n)}^{n}\right\}$ of $[0,1]$ consisting of half-open intervals of the same length, with $\left|I_{j}^{n}\right|<\delta_{n}$ for all $j$, and set $A_{j}^{n+1}:=B_{n} \cap I_{j}^{n}$ whenever the intersection is non-negligible. From (2), we see that 


$$
\left\|f\left(t_{j}^{n+1}\right)-\frac{\int_{A_{j}^{n+1}} f}{\lambda\left(A_{j}^{n+1}\right)}\right\| \leq \varepsilon
$$

holds true, for every $n$ and every choice of points $t_{j}^{n+1} \in A_{j}^{n+1}$. Finally, since the sequence $\left(A_{j}^{n+1}\right)_{\{n \in \mathbb{N}, j \in\{1, \ldots, N(n)\}\}}$ is composed by pairwise disjoint sets, we can rearrange them in a sequence $\left(A_{h}\right)_{h=2}^{\infty}$. Adding also the null set $A_{1}:=[0,1] \backslash\left(\cup_{h=2}^{\infty} A_{h}\right)$, we claim that the partition $\left(A_{h}\right)_{h}$ obtained in this way is the requested one.

Indeed, let $\left\{A_{k}^{\prime}\right\}_{k}$ be any refinement of $\left(A_{h}\right)_{h}$. Since each non-negligible $A_{k}^{\prime}$ is contained in some set of the type $B_{n} \cap I_{j}$, we also have, similarly to (2)

$$
\left\|f\left(t_{k}\right)-\frac{\int_{A_{k}^{\prime}} f}{\lambda\left(A_{k}^{\prime}\right)}\right\| \leq \varepsilon
$$

as soon as $t_{k} \in A_{k}^{\prime}$. So

$$
\begin{aligned}
& \sum_{k}\left\|f\left(t_{k}\right) \lambda\left(A_{k}^{\prime}\right)-\int_{A_{k}^{\prime}} f\right\| \leq \sum_{n} \sum_{j} \sum_{A_{k}^{\prime} \subset B_{n} \cap I_{j}^{n}}\left\|f\left(t_{k}\right) \lambda\left(A_{k}^{\prime}\right)-\int_{A_{k}^{\prime}} f\right\| \\
& \leq \sum_{n} \sum_{j} \sum_{A_{k}^{\prime} \subset B_{n} \cap I_{j}^{n}} \varepsilon \lambda\left(A_{k}^{\prime}\right)=\sum_{n} \sum_{j} \varepsilon \lambda\left(B_{n} \cap I_{j}^{n}\right)=\sum_{n} \varepsilon \lambda\left(B_{n}\right)=\varepsilon,
\end{aligned}
$$

as requested.

Remark 3.9 We observe that if $f$ satisfies (1), in general $f$ is not variationally Henstock integrable: indeed, in [6, Remark 4.3] it is shown that for every Banach space $X$ there are Pettis integrable mappings $f:[0,1] \rightarrow X$ whose variational measure associated to the integral fails to be $\lambda$-continuous. In particular, if $X$ is separable, such functions are also Birkhoff integrable and strongly measurable.

Corollary 3.10 Assume that $f:[0,1] \rightarrow X$ is strongly measurable. Then $f$ is Birkhoff integrable if and only if there exists a countable partition $\left(A_{k}\right)_{k}$ of $[0,1]$ such that the restriction $\left.f\right|_{A_{k}}$ is bounded whenever $\lambda\left(A_{k}\right)>0$ and $\left\{\sum_{k} f\left(t_{k}\right) \lambda\left(A_{k}\right), t_{k} \in A_{k}\right\}$ is made up of unconditionally convergent series.

Proof It is enough to apply Proposition 3.8 together with [14, Proposition 2.2].

By the properties of the Rådström embedding we can obtain also that a Bochner measurable multifunction $\Gamma:[0,1] \rightarrow c w k(X)$ is Birkhoff integrable if and only is there exists a countable partition of $[0,1]:\left(A_{k}\right)_{k}$ such that the restriction $\left.F\right|_{A_{k}}$ is bounded whenever $\lambda\left(A_{k}\right)>0$ and $\left\{\sum_{k} \Gamma\left(t_{k}\right) \lambda\left(A_{k}\right), t_{k} \in A_{k}\right\}$ is made up of unconditionally convergent series.

Finally, we would like to remark that, for strongly measurable maps, Birkhoff integrability can be labeled in a form that recalls the notion of variational Henstock integrability (though there is no direct implication between the two notions, in general). We first give a definition. 
Definition 3.11 Given a function $f:[0,1] \rightarrow X$, we say that $f$ is strongly Birkhoff integrable if there exists a $\lambda$-continuous $\sigma$-additive measure $\phi: \mathcal{L} \rightarrow X$ such that, for every $\varepsilon>0$ it is possible to find a countable partition $P$ of $[0,1]$, made with measurable sets, such that, as soon as $\left\{A_{n}\right\}_{n}$ is a refinement of $P$, it holds

$$
\sum_{n}\left\|f\left(t_{n}\right) \lambda\left(A_{n}\right)-\phi\left(A_{n}\right)\right\| \leq \varepsilon
$$

for every choice of $t_{n} \in A_{n}, n \in \mathbb{N}$.

We now observe that, as soon as $f:[0,1] \rightarrow X$ is strongly measurable and Birkhoff integrable, it turns out to be also strongly Birkhoff integrable, thanks to Proposition 3.8, and of course $\phi$ is the (Birkhoff) integral function of $f$.

Conversely, we have the following results.

Proposition 3.12 Assume that $f:[0,1] \rightarrow X$ is strongly Birkhoff integrable. Then it is also Birkhoff integrable.

Proof Fix $\varepsilon>0$, and let $P:=\left\{E_{j}: j \in \mathbb{N}\right\}$ be the corresponding partition in the definition of strong Birkhoff integrability. Choose now any finer partition $\left\{A_{k}\right\}_{k}$, set $x:=\sum_{k} \phi\left(E_{k}\right)=\phi([0,1])$, and observe that there exists an integer $N$ such that $\left\|x-\sum_{k \leq n} \phi\left(A_{k}\right)\right\| \leq \varepsilon$ for every $n \geq N$. So, for every $n \geq N$, and every choice of points $t_{k} \in A_{k}$, we have

$$
\begin{aligned}
& \left\|\sum_{k=1}^{n} f\left(t_{k}\right) \lambda\left(A_{k}\right)-x\right\| \leq\left\|\sum_{k=1}^{n} f\left(t_{k}\right) \lambda\left(A_{k}\right)-\sum_{k=1}^{n} \phi\left(A_{k}\right)\right\|+\varepsilon \\
& \quad \leq \sum_{k=1}^{n}\left\|f\left(t_{k}\right) \lambda\left(A_{k}\right)-\phi\left(A_{k}\right)\right\|+\varepsilon \leq \sum_{k=1}^{\infty}\left\|f\left(t_{k}\right) \lambda\left(A_{k}\right)-\phi\left(A_{k}\right)\right\|+\varepsilon \leq 2 \varepsilon .
\end{aligned}
$$

This means that

$$
\underset{n}{\lim \sup _{n}}\left\|\sum_{k=1}^{n} f\left(t_{k}\right) \lambda\left(A_{k}\right)-x\right\| \leq 2 \varepsilon .
$$

This property in [9] is called simple-Birkhoff integrability, and is proved in [9, Theorem 3.18] to be equivalent to Birkhoff integrability of $f$.

Proposition 3.13 Assume that $f:[0,1] \rightarrow X$ is strongly Birkhoff integrable. Then $f$ is also strongly measurable.

Proof We shall follow the technique of [19, Lemma 3]. First of all, let us denote by $V$ the separable subspace of $X$ generated by the range of $\phi$. Next, for every $\varepsilon>0$ let us set

$$
T_{\varepsilon}:=\left\{t \in[0,1]: d_{H}(f(t), V)>\varepsilon\right\} .
$$


Now, we shall prove that $\lambda\left(T_{\varepsilon}\right)=0$ for all $\varepsilon>0$, from which it will follow that $f$ is separably-valued, and so also strongly measurable, thanks to the Pettis Theorem. So, assume by contradiction that there exists a $\varepsilon^{\prime}>0$ such that $\lambda^{*}\left(T_{\varepsilon^{\prime}}\right)=a>0$. Let $A^{\prime}$ denote any measurable set such that $T_{\mathcal{E}^{\prime}} \subset A^{\prime}$ and $\lambda\left(A^{\prime}\right)=\lambda^{*}\left(T_{\mathcal{E}^{\prime}}\right)=a$.

Now, fix $0<\varepsilon<\varepsilon^{\prime}$ and take any partition $\Pi \equiv\left\{E_{j}\right\}_{j}$ of measurable sets satisfying the condition of strong Birkhoff integrability of $f$ with respect to $a \varepsilon$. Moreover let $\left\{A_{n}\right\}_{n}$ be any finer partition, such that $A^{\prime} \cap E_{j}$ and $E_{j} \backslash A^{\prime}$ are among the sets $A_{n}$. Without loss of generality, we can assume that $\lambda\left(A_{n}\right)>0$ for all $n$. Observe that, if $A_{n}$ is of the type $A^{\prime} \cap E_{j}$, then $A_{n} \cap T_{\mathcal{E}^{\prime}}$ is non-empty, otherwise $A^{\prime} \backslash A_{n}$ would contain $T_{\mathcal{E}^{\prime}}$ but would have smaller measure than $a$. So, we can choose $t_{n} \in A_{n} \cap T_{\mathcal{E}^{\prime}}$ as soon as $A_{n}$ is of the type $A^{\prime} \cap E_{j}$. Thus we have

$$
\sum_{n}^{\prime}\left\|f\left(t_{n}\right) \lambda\left(A_{n}\right)-\phi\left(A_{n}\right)\right\| \leq a \varepsilon
$$

where the $\sum^{\prime}$ runs along the sets $A_{n}$ of the type $A^{\prime} \cap E_{j}$. Therefore, since $A^{\prime}$ is the union of all the sets $A_{n}$ of the type above,

$$
\sum_{n}^{\prime}\left\|f\left(t_{n}\right) \lambda\left(A_{n}\right)-\phi\left(A_{n}\right)\right\| \leq \varepsilon \sum_{n}^{\prime} \lambda\left(A_{n}\right)
$$

i.e.

$$
\sum_{n}^{\prime}\left\|f\left(t_{n}\right)-\frac{\phi\left(A_{n}\right)}{\lambda\left(A_{n}\right)}\right\| \lambda\left(A_{n}\right) \leq \varepsilon \sum_{n}^{\prime} \lambda\left(A_{n}\right) .
$$

This inequality implies that, for at least one integer $n$, it is

$$
\left\|f\left(t_{n}\right)-\frac{\phi\left(A_{n}\right)}{\lambda\left(A_{n}\right)}\right\| \leq \varepsilon<\varepsilon^{\prime},
$$

but this is impossible, since $\frac{\phi\left(A_{n}\right)}{\lambda\left(A_{n}\right)} \in H$ and $t_{n} \in T_{\varepsilon^{\prime}}$. This proves that $\lambda\left(T_{\varepsilon}\right)=0$ for every $\varepsilon>0$, and so $f$ is strongly measurable.

Summarizing, we get the following conclusion.

Theorem 3.14 Assume that $f:[0,1] \rightarrow X$ is any mapping. Then $f$ is strongly Birkhoff integrable if and only if it is strongly measurable and Birkhoff integrable.

\section{References}

1. Birkhoff, G.: Integration of functions with values in a Banach space. Trans. Am. Math. Soc. 38, 357-378 (1935)

2. Boccuto, A., Sambucini, A.R.: A note on comparison between Birkhoff and McShane-type integrals for multifunctions. Real Anal. Exch. 37(2), 315-324 (2012) 
3. Boccuto, A., Minotti, A.M., Sambucini, A.R.: Set-valued Kurzweil-Henstock integral in Riesz space setting. PanAmerican Math. J. 23(1), 57-74 (2013)

4. Boccuto, A., Candeloro, D., Sambucini, A.R.: Henstock multivalued integrability in Banach lattices with respect to pointwise non atomic measures. Atti Accad Naz. Lincei Rend. Lincei Mat. Appl. 26(4), 363-383 (2015). https://doi.org/10.4171/RLM/710

5. Bongiorno, B., Di Piazza, L., Musiał, K.: Approximation of Banach space valued non-absolutely integrable functions by step functions. Glasg. Math. J. 50, 583-593 (2008)

6. Bongiorno, B., Di Piazza, L., Musiał, K.: A variational Henstock integral characterization of the Radon-Nikodym Property. Ill. J. Math. 53(1), 87-99 (2009)

7. Candeloro, D., Sambucini, A.R.: Order-type Henstock and Mc Shane integrals in Banach lattices setting. In: Sisy 20014- IEEE 12th International Symposium on Intelligent Systems and Informatics, Subotica-Serbia; 09/2014 (2014). https://doi.org/10.1109/SISY.2014.6923557

8. Candeloro, D., Sambucini, A.R.: Comparison between some norm and order gauge integrals in Banach lattices. PanAmerican Math. J. 25(3), 1-16 (2015)

9. Candeloro, D., Croitoru, A., Gavrilut, A., Sambucini, A.R.: An extension of the Birkhoff integrability for multifunctions. Mediter. J. Math. 13(5), 2551-2575 (2016). https://doi.org/10.1007/s00009-0150639-7

10. Candeloro, D., Di Piazza, L., Musial, K., Sambucini, A.R.: Gauge integrals and selections of weakly compact valued multifunctions. J. Math. Anal. Appl. 441(1), 293-308 (2016). https://doi.org/10.1016/ j.jmaa.2016.04.009

11. Candeloro, D., Di Piazza, L., Musial, K., Sambucini, A.R.: Relations among gauge and Pettis integrals for multifunctions with weakly compact convex values. Ann. Mat. 197(1), 171-183 (2018). https:// doi.org/10.1007/s10231-017-0674-z

12. Caponetti, D., Marraffa, V., Naralenkov, K.: On the integration of Riemann-measurable vector-valued functions. Monatsh. Math. 182(3), 513-586 (2017). https://doi.org/10.1007/s00605-016-0923-z

13. Cascales, B., Rodríguez, J.: Birkhoff integral for multi-valued functions. J. Math. Anal. Appl. 297(2), 540-560 (2004)

14. Cascales, B., Rodríguez, J.: The Birkhoff integral and the property of Bourgain. Math. Ann. 331(2), 259-279 (2005)

15. Cascales, C., Kadets, V., Rodríguez, J.: The Pettis integral for multi-valued functions via single-valued ones. J. Math. Anal. Appl. 332(1), 1-10 (2007)

16. Cascales, C., Kadets, V., Rodríguez, J.: Measurable selectors and set-valued Pettis integral in nonseparable Banach spaces. J. Funct. Anal. 256, 673-699 (2009)

17. Castaing, C., Valadier, M.: Convex Analysis and Measurable Multifunctions, Lecture Notes in Mathematics, vol. 580. Springer, Berlin (1977)

18. Di Piazza, L.: Variational measures in the theory of the integration in $R^{m}$. Czechoslov. Math. J. 51(1), 95-110 (2001)

19. Di Piazza, L., Musiał, K.: A characterization of variationally McShane integrable banach-space valued functions. Ill. J. Math. 45(1), 279-289 (2001)

20. Di Piazza, L., Musiał, K.: Set-valued Henstock-Kurzweil-Pettis integral. Set Valued Anal. 13, 167-179 (2005)

21. Di Piazza, L., Musiał, K.: A decomposition of Denjoy-Khintchine-Pettis and Henstock-KurzweilPettis integrable multifunctions. In: Curbera, G.P., Mockenhaupt, G., Ricker, W.J. (eds.) Vector Measures, Integration and Related Topics, Operator Theory: Advances and Applications, vol. 201, pp. 171-182. Birkhauser Verlag, Basel (2010)

22. Di Piazza, L., Musiał, K.: Relations among Henstock, McShane and Pettis integrals for multifunctions with compact convex values. Monatsh. Math. 173(4), 459-470 (2014)

23. Di Piazza, L., Porcello, G.: Radon-Nikodym theorems for finitely additive multimeasures. Z. Anal. ihre. Anwend. (ZAA) 34(4), 373-389 (2015). https://doi.org/10.4171/ZAA/1545

24. Fremlin, D.H.: The Henstock and McShane integrals of vector-valued functions. Ill. J. Math. 38(3), 471-479 (1994)

25. Fremlin, D.H., Mendoza, J.: On the integration of vector-valued functions. Ill. J. Math. 38, 127-147 (1994)

26. Gámez, J.L., Mendoza, J.: On Denjoy-Dunford and Denjoy-Pettis integrals. Stud. Math. 130(2), 115133 (1998)

27. Gordon, R.A.: The Integrals of Lebesgue, Denjoy, Perron and Henstock. Graduate Studies in Mathematics, vol. 4. AMS, Providence (1994) 
28. Hu, S., Papageorgiou, N.S.: Handbook of Multivalued Analysis I and II, Mathematics and Its Applications, vol. 419. Kluwer Academic Publisher, Dordrecht (1997)

29. Labuschagne, C.C.A., Marraffa, V.: Operator martingale decompositions and the Radon-Nikodym property in Banach spaces. J. Math. Anal. Appl. 363(2), 357-365 (2010). https://doi.org/10.1016/j. jmaa.2009.08.054

30. Labuschagne, C.C.A., Pinchuck, A.L., van Alten, C.J.: A vector lattice version of Rådström's embedding theorem. Quaest. Math. 30(3), 285-308 (2007)

31. Marraffa, V.: The Birkhoff integral and the Bourgain property in a locally convex space. Real Anal. Exch. 32(2), 1-19 (2006/2007)

32. Musiał, K.: Topics in the theory of Pettis integration. Rend. Istit. Mat. Univ. Trieste 23, 177-262 (1991)

33. Musiał, K.: Pettis integral. In: Handbook of Measure Theory I, North-Holland, Amsterdam, pp. 531586 (2002)

34. Musiał, K.: Pettis integrability of multifunctions with values in arbitrary Banach spaces. J. Convex Anal. 18(3), 769-810 (2011)

35. Musiał, K.: Approximation of Pettis integrable multifunctions with values in arbitrary Banach spaces. J. Convex Anal. 20(3), 833-870 (2013)

36. Naralenkov, K.M.: A Lusin type measurability property for vector-valued functions. J. Math. Anal. Appl. 417(1), 293-307 (2014). https://doi.org/10.1016/j.jmaa.2014.03.029

37. Pettis, B.J.: On integration in vector spaces. Trans. Am. Math. Soc. 44(2), 277-304 (1938)

38. Porcello, G.: Multimeasures and integration of multifunctions in Banach spaces. In: Dottorato di Ricerca in Matematica e Informatica XXIV ciclo, University of Palermo (Italy).https://iris.unipa.it/ retrieve/handle/10447/91026/99048/TesiDottoratoGiovanniPorcello.pdf (2014)

39. Potyrala, M.: The Birkhoff and Variationally McShane integrals of vector valued functions. Folia Math. Acta Univ. Lodz. 13(1), 31-39 (2006)

40. Potyrala, M.: Some remarks about Birkhoff and Riemann-Lebesgue integrability of vector valued functions. Tatra Mt. Math. Publ. 35, 97-106 (2007) 